TIP Periodica Polytechnica

Transportation Engineering

42(1), pp. 37-42, 2014

DOI:10.3311/PPtr. 7183

http://www.pp.bme.hu/tr/article/view/7183

Creative Commons Attribution (i)

RESEARCH ARTICLE

\section{Theoretical investigation of emission and delay based intersection controlling and synchronizing in Budapest}

Ferenc Meszaros / Adam Torok

RECEIVED 8 November 2013; ACCEPTEd 14 January 2014

\section{Abstract}

Road transport is one of the main land transport modes providing flexible door to door services. New type of control of road traffic flows in urban intersections is modelled in this article. Furthermore the synchronizing possibilities of intersections are investigated as well. Cost of $\mathrm{CO}_{2}, \mathrm{CO}, \mathrm{CH}, \mathrm{NO}_{x}, \mathrm{PM}$ and value of travel time had been used by the authors in order to estimate the cost of road users as a basis of control. The article presents advice for optimal control and gives simulation results based on the emission and delay based costing. Traffic flow parameters, such as traffic flow concentration and traffic flow speed are presented based on real traffic data of investigated intersections. In this article not only single intersections were investigated, but a chain of intersections in order to analyze the recovery potential of synchronization reserves.

\section{Keywords \\ intersection controlling · cost function · synchronizing}

\section{Ferenc Meszaros}

Department of Transport Technology and Transport Economics, Budapest University of Technology and Economics,

Bertalan L. u. 2., H-1111 Budapest, Hungary

e-mail: fmeszaros@kgazd.bme.hu

\section{Adam Torok}

Department of Transport Technology and Transport Economics, Budapest University of Technology and Economics,

Bertalan L. u. 2., H-1111 Budapest, Hungary

e-mail: atorok@kgazd.bme.hu

\section{Introduction}

Vehicle flows carry people, distribute industrial freight and work equipment on road network elements (Torok, Berta, 2010). Majority of these road vehicles are driven by internal combustion engines; therefore besides practical use they also create a lot of problems, such as air pollution and particulate matter by combustion products, noise and vibration. Various problems caused by vehicles are discussed in the article written by Makaras (Makaras, et. al., 2011). Wang (Wang. et. al., 2008) presented various methods of fuel consumption and engines emission measuring as well as coefficients of efficiency. Szendro (Szendro, et. al., 2012) investigated climate fluctuation changes and energy consumption in Hungary. Smit (Smit, et. al., 2008) presented and generalize three emission models, where the impact of congestions on motor vehicles' emission is evaluated differently and present indicators to identify transport congestions. Jakimavičius, and Burinskienè (Jakimavičius, M.; Burinskienè M., 2010) investigated vehicle flow optimization methods and their application possibilities when informing traffic users about the situation in the city. Signal control is a traditional method to improve traffic efficiency at intersection areas, and the related signal design problems have been investigated for several decades. According to the traffic flow state, two categories of signal design problems are addressed so far: static-flow-based problems and dynamic-flow-based problems (Ren et. al., 2013). In order to define the level of service of intersections, it is necessary to know some of the basic parameters of traffic flow, like flow intensity, vehicles velocity and density (Bogdanović et. al., 2013). Social cost intersection controlling is an up-to-date research topic as it could increase the level of intersection (Meszaros, Markovits-Somogyi, Bokor, 2012). This article gives an example of applying models of traffic controlling in the basis of emission and delay based controlling and can be a solid base of further tolling development (Torok, Siposs, Meszaros, 2011). The article not only investigates one intersection, but the possibility of synchronising the controlled intersections. 


\section{Methodology}

An intersection can be characterized by directions and lanes. When describing traffic flows, a traffic lane is used as a keyword. An assumption is taken that cars cannot drive on an opposite traffic lane; therefore, the road is split into separate traffic lanes and two-way roads are described in the mathematical model as a separate one-way road with one or several traffic lanes (Junevičius, Bogdevičius, Torok, 2011). In this model a traffic lane segment is taken as a finite-length line that ends in the intersection. Traffic flow was measured and emission and delay based cost was calculated as follows (1), (2):

$$
\operatorname{TTS}_{i}=\sum_{k=1}^{P}\left(v_{i, k} \cdot \tau_{i, k}\right)
$$

where,

TTS $_{\mathrm{i}}$ : Estimated cost or revenue of travel time saving by direction i. [HUF]

$\mathrm{v}_{\mathrm{i}, \mathrm{j}}$ : Value of travel time for passenger $\mathrm{k}$ at direction i. [HUF/s]

$\tau_{\mathrm{i}, \mathrm{k}}: \quad$ Waiting time in the lane at direction i. [s]

$$
E C_{i}=\sum_{l=1}^{r} \sum_{j=1}^{m}\left(\varepsilon_{i, k} \cdot \tau_{i, k} \cdot \rho_{l}\right)
$$

where,

$\mathrm{EC}_{\mathrm{i}}$ : Estimated Environmental Cost of direction i. [HUF];

$\varepsilon_{\mathrm{i}, \mathrm{j}}$ : Environmental emission factor of vehicle category $\mathrm{j}$ in directory i. [g/s] (Csikos, Varga, 2011), (Zoldy, 2011), (Bereczky, 2012), (Barabas, Todorut, 2011),

(Negoiţescu, Tokar, 2013), (Makarevičiene, et. al., 2013);

$\tau_{\mathrm{i}, \mathrm{k}}: \quad$ Waiting time in the lane at direction i. [s] (Gal, 2012);

$\rho_{\mathrm{i}, 1}$ : Cost of environmental emission for pollutant 1 [HUF/g] (Tánczos, Bokor, 2004)

For modelling purposes not only the detailed plan of each intersection was available but the plan of signalling as well (Fig. 1).

Authors have conducted a traffic measurement of each intersection in peak-time in order to estimate the traffic related social costs.

\section{Results}

\subsection{Place 1: Gellert sqr}

Authors firstly determined the optimal green time for intersection (place 1: Gellert sqr.) in case of minimizing delay (1), (Tab. 1.). For the optimal green times the total cost was determined (Tab. 2.). Authors determined the optimal green time for intersection (place 1: Gellert sqr.) in case of minimizing environmental pollution (2), (Tab. 3.): For the optimal green times the total cost was determined (Table 4.)

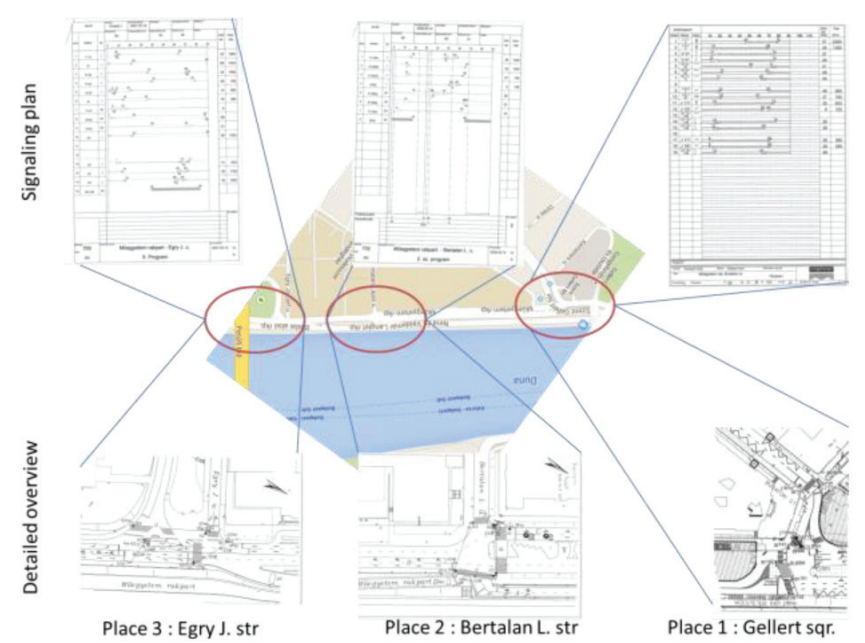

Fig. 1. Schematic overview of intersections and data availability

Tab. 1. The current and optimal (minimized delay) green times

\begin{tabular}{cccc}
\hline & $\begin{array}{c}\text { Direction 1: } \\
\text { from } \\
\text { Budafoki } \\
\text { str }\end{array}$ & $\begin{array}{c}\text { Direction 2: } \\
\text { from } \\
\text { Gellert } \\
\text { sqr }\end{array}$ & $\begin{array}{c}\text { Direction 3: } \\
\text { from } \\
\text { Múegyetem } \\
\text { quay }\end{array}$ \\
\hline $\begin{array}{c}\text { Current green time [s] } \\
\text { Optimal in case } \\
\text { of minimal delays [s] }\end{array}$ & 30 & 49 & 37 \\
\hline
\end{tabular}

\begin{tabular}{|c|c|c|c|c|}
\hline & & $\begin{array}{c}\text { Direction 1: } \\
\text { from } \\
\text { Budafoki } \\
\text { str }\end{array}$ & $\begin{array}{l}\text { Direction 2: } \\
\quad \text { from } \\
\text { Gellert sqr }\end{array}$ & $\begin{array}{l}\text { Direction 3: } \\
\text { from } \\
\text { Múegyetem } \\
\text { quay }\end{array}$ \\
\hline \multirow{4}{*}{$\begin{array}{l}\text { Travel } \\
\text { time } \\
\text { delay } \\
\text { cost } \\
\text { of current } \\
\text { signalling } \\
\text { [HUF/h] }\end{array}$} & $\begin{array}{c}\text { Passenger } \\
\text { Car }\end{array}$ & 81684 & 19772 & 20642 \\
\hline & BUS & 43635 & 17909 & - \\
\hline & $\begin{array}{l}\text { sum of } \\
\text { direction }\end{array}$ & 125319 & 37681 & 20642 \\
\hline & total sum & & 183642 & \\
\hline \multirow{4}{*}{$\begin{array}{c}\text { Travel } \\
\text { time } \\
\text { delay } \\
\text { cost of } \\
\text { new } \\
\text { signalling } \\
\text { [HUF/h] }\end{array}$} & $\begin{array}{c}\text { Passenger } \\
\text { Car }\end{array}$ & 57517 & 31802 & 29268 \\
\hline & BUS & 34483 & 24539 & - \\
\hline & $\begin{array}{l}\text { sum of } \\
\text { direction }\end{array}$ & 92000 & 56341 & 29268 \\
\hline & total sum & & 177609 & \\
\hline
\end{tabular}

Tab. 2. Cost of delay

Tab. 3. The current and optimal (minimized environmental pollution) green times

\begin{tabular}{cccc}
\hline & $\begin{array}{c}\text { Direction 1: } \\
\text { from } \\
\text { Budafoki } \\
\text { str }\end{array}$ & $\begin{array}{c}\text { Direction 2: } \\
\text { from } \\
\text { Gellert } \\
\text { sqr }\end{array}$ & $\begin{array}{c}\text { Direction 3: } \\
\text { from } \\
\text { Múegyetem } \\
\text { quay }\end{array}$ \\
\hline $\begin{array}{c}\text { Current green time [s] } \\
\text { Optimal in case } \\
\text { of minimal delays [s] }\end{array}$ & 30 & 49 & 37 \\
\hline
\end{tabular}


Tab. 4. Cost of environmental emission

\begin{tabular}{|c|c|c|c|c|}
\hline & & $\begin{array}{c}\text { Direction 1: } \\
\text { from } \\
\text { Budafoki } \\
\text { str }\end{array}$ & $\begin{array}{c}\text { Direction 2: } \\
\text { from } \\
\text { Gellert sqr }\end{array}$ & $\begin{array}{c}\text { Direction } 3: \\
\text { from } \\
\text { Múegyetem } \\
\text { quay }\end{array}$ \\
\hline \multirow{4}{*}{$\begin{array}{c}\text { Environ- } \\
\text { mental } \\
\text { emission } \\
\text { cost of } \\
\text { current } \\
\text { signalling } \\
\text { [HUF/h] }\end{array}$} & $\begin{array}{c}\text { Passenger } \\
\text { Car }\end{array}$ & 196.91 & 47.56 & 50.17 \\
\hline & $\begin{array}{l}\text { Goods } \\
\text { Vehicle }\end{array}$ & 9.57 & 2.32 & 1.16 \\
\hline & $\begin{array}{l}\text { sum of } \\
\text { direction }\end{array}$ & 206.19 & 49.88 & 51.33 \\
\hline & total sum & & 307.69 & \\
\hline \multirow{4}{*}{$\begin{array}{c}\text { Environ- } \\
\text { mental } \\
\text { emission } \\
\text { cost of } \\
\text { new } \\
\text { signalling } \\
\text { [HUF/h] }\end{array}$} & $\begin{array}{c}\text { Passenger } \\
\text { Car }\end{array}$ & 147.61 & 70.76 & 67.57 \\
\hline & $\begin{array}{l}\text { Goods } \\
\text { Vehicle }\end{array}$ & 7.25 & 3.48 & 1.45 \\
\hline & $\begin{array}{l}\text { sum of } \\
\text { direction }\end{array}$ & 154.57 & 74.24 & 69.02 \\
\hline & total sum & & 297.83 & \\
\hline \multirow{2}{*}{$\begin{array}{c}\text { Change } \\
\text { [\%] }\end{array}$} & $\begin{array}{l}\text { sum of } \\
\text { direction }\end{array}$ & $-25.08 \%$ & $48.81 \%$ & $34.50 \%$ \\
\hline & & & $-3.14 \%$ & \\
\hline
\end{tabular}

Tab. 5. The current and optimal (minimized environmental pollution) green times

\begin{tabular}{cccc}
\hline & $\begin{array}{c}\text { Direction 1: } \\
\text { from } \\
\text { Petöfi } \\
\text { bridge }\end{array}$ & $\begin{array}{c}\text { Direction 2: } \\
\text { from } \\
\text { Gellert } \\
\text { sqr }\end{array}$ & $\begin{array}{c}\text { Direction 3: } \\
\text { from } \\
\text { Alsorakpart }\end{array}$ \\
\hline $\begin{array}{c}\text { Current green time [s] } \\
\text { Optimal in case } \\
\text { of minimal delays [s] }\end{array}$ & 35 & 37 & 38 \\
\hline
\end{tabular}

\subsection{Place 2: Bertalan L. str.}

The same methodology was used to calculate the costs and green times related to Place 2: Bertalan L. str (1), (Tab. 5.). For the optimal green times the total cost was determined (Tab 6.). Authors determined the optimal green time for intersection (place 2: Bertalan Str.) in case of minimising environmental pollution (2), (Tab. 7.). As it can be seen in Tab. 7 the same result was find as in case of delay minimising. It can be easily understandable as there are no buses or goods vehicles in the traffic flow. For the optimal green times the total cost was determined (Tab. 8.).
Tab. 6. Cost of delay

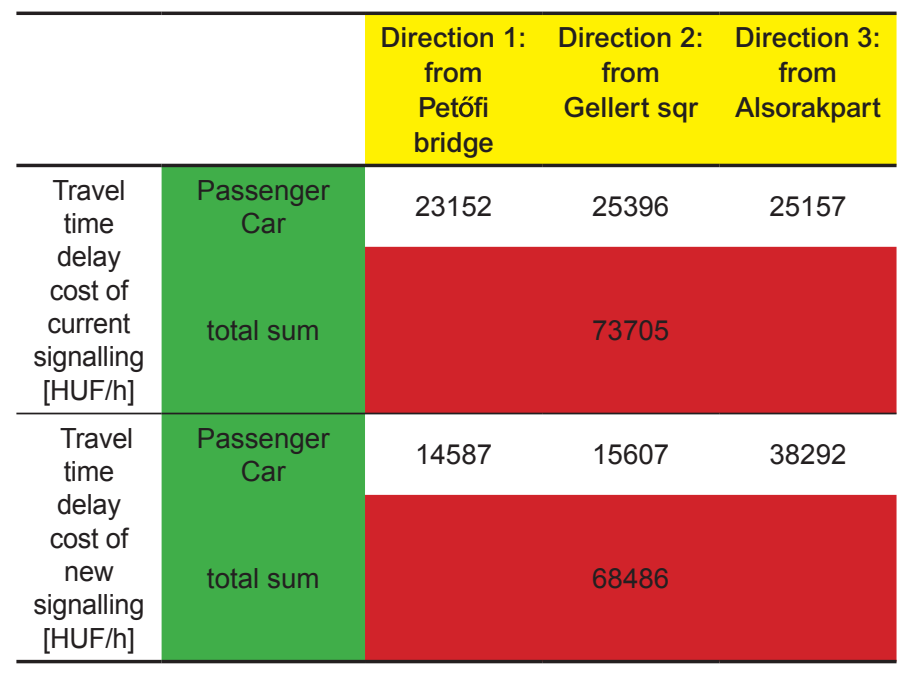

Tab. 7. The current and optimal (minimized environmental pollution) green times

\begin{tabular}{cccc}
\hline & $\begin{array}{c}\text { Direction 1: } \\
\text { from } \\
\text { Petöfi } \\
\text { bridge }\end{array}$ & $\begin{array}{c}\text { Direction 2: } \\
\text { from } \\
\text { Gellert } \\
\text { sqr }\end{array}$ & $\begin{array}{c}\text { Direction 3: } \\
\text { from } \\
\text { Alsorakpart }\end{array}$ \\
\hline $\begin{array}{c}\text { Current green time [s] } \\
\text { Optimal in case } \\
\text { of minimal delays [s] }\end{array}$ & 35 & 37 & 38 \\
\hline
\end{tabular}

Tab. 8. Cost of environmental emission

\begin{tabular}{|c|c|c|c|c|}
\hline & & $\begin{array}{c}\text { Direction 1: } \\
\text { from } \\
\text { Petőfi } \\
\text { bridge }\end{array}$ & $\begin{array}{l}\text { Direction 2: } \\
\text { from } \\
\text { Gellert sqr }\end{array}$ & $\begin{array}{c}\text { Direction 3: } \\
\text { from } \\
\text { Alsorakpart }\end{array}$ \\
\hline \multirow{4}{*}{$\begin{array}{l}\text { Environ- } \\
\text { mental } \\
\text { emission } \\
\text { cost of } \\
\text { current } \\
\text { signalling } \\
\text { [HUF/h] }\end{array}$} & $\begin{array}{c}\text { Passenger } \\
\text { Car }\end{array}$ & 56.26 & 61.77 & 60.9 \\
\hline & $\begin{array}{l}\text { Goods } \\
\text { Vehicle }\end{array}$ & 1.45 & 1.45 & 2.03 \\
\hline & $\begin{array}{l}\text { sum of } \\
\text { direction }\end{array}$ & 57.71 & 63.22 & 62.93 \\
\hline & total sum & & 183.86 & \\
\hline \multirow{4}{*}{$\begin{array}{l}\text { Environ- } \\
\text { mental } \\
\text { emission } \\
\text { cost of } \\
\text { new } \\
\text { signalling } \\
\text { [HUF/h] }\end{array}$} & $\begin{array}{c}\text { Passenger } \\
\text { Car }\end{array}$ & 35.38 & 37.99 & 92.8 \\
\hline & $\begin{array}{l}\text { Goods } \\
\text { Vehicle }\end{array}$ & 0.87 & 0.87 & 3.19 \\
\hline & $\begin{array}{l}\text { sum of } \\
\text { direction }\end{array}$ & 36.25 & 38.86 & 95.99 \\
\hline & total sum & & 171.1 & \\
\hline \multirow{2}{*}{$\begin{array}{c}\text { Change } \\
{[\%]}\end{array}$} & $\begin{array}{l}\text { sum of } \\
\text { direction }\end{array}$ & $-36.99 \%$ & $-38.55 \%$ & $52.21 \%$ \\
\hline & & & $-6.95 \%$ & \\
\hline
\end{tabular}




\subsection{Place 3: Egry J. str.}

The same methodology was used to calculate the costs and green times related to Place 3: Egry J. str, (1), (Tab. 9.). For the optimal green times the total cost was determined (Tab. 10.). Authors determined the optimal green time for intersection (place 3: Irinyi J. str) in case of minimising environmental pollution (2), (Tab 11.). For the optimal green times the total cost was determined (Tab. 12.).

\section{Analysis}

After analyzing the intersections separately the authors have investigated the possibility of synchronizing of basis of delay minimizing or minimizing environmental pollution. Due to the same optimal solution for Place 2: Bertalan L. str and Place 3: Egry J. str the synchronizing can be easily done in both case (See Fig. 2 and Fig. 3.).

\section{Conclusions}

In this article we have introduced the social cost based intersection synchroning as a form of network controlling. As the single programmed intersections can be grouped to chains and can be synchronized the same algorithm can be derived for more complex social cost based intersections (Fig. 4.). As it has been shown the social interest would lead to different optimum in case of intersection controlling compared to single program (traffic) controlled situation. Further reserved potential can be recovered with synchroning these social cost controlled intersections.

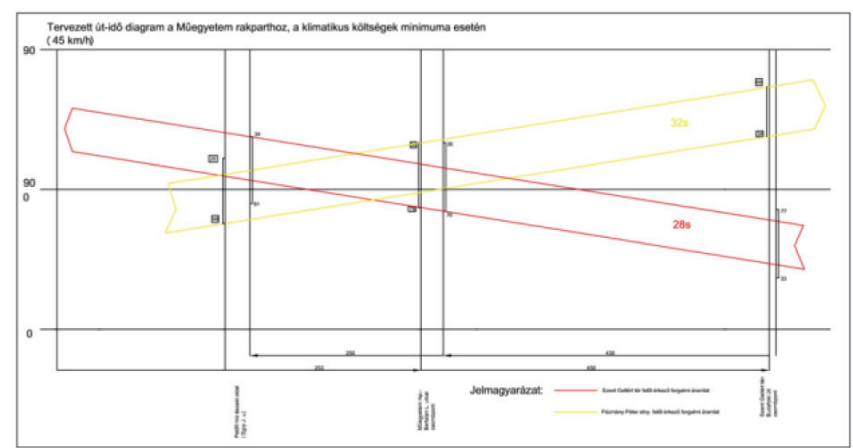

Fig. 2. Delay minimized synchronizing

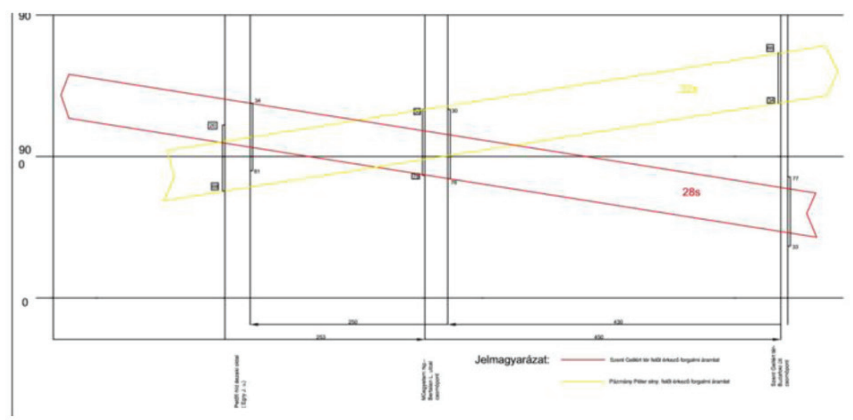

Fig. 3. Emission minimized synchronizing
Tab. 9. The current and optimal (minimized delay) green times

\begin{tabular}{|c|c|c|c|c|}
\hline & $\begin{array}{c}\text { Direction } \\
1: \\
\text { from } \\
\text { Petőfi } \\
\text { bridge }\end{array}$ & $\begin{array}{c}\text { Direction } \\
2 \text { : } \\
\text { from } \\
\text { Gellert } \\
\text { sqr }\end{array}$ & $\begin{array}{c}\text { Direction } \\
\text { 3: } \\
\text { from } \\
\text { Irinyi J. } \\
\text { str }\end{array}$ & $\begin{array}{c}\text { Direction } \\
4: \\
\text { from } \\
\text { Egry J. } \\
\text { str }\end{array}$ \\
\hline $\begin{array}{l}\text { Current green } \\
\text { time [s] }\end{array}$ & 33 & 34 & 19 & 14 \\
\hline $\begin{array}{l}\text { Theore-tical case of } \\
\text { minimal delays }[\mathrm{s}]^{*}\end{array}$ & 45 & 46 & 7 & 2 \\
\hline $\begin{array}{c}\text { Optimal in case } \\
\text { of minimal delays }[\mathrm{s}]\end{array}$ & 42 & 43 & 10 & 5 \\
\hline
\end{tabular}

Tab. 10. Cost of delay

\begin{tabular}{|c|c|c|c|c|c|}
\hline & & $\begin{array}{c}\text { Direction } \\
1: \\
\text { from } \\
\text { Petőfi } \\
\text { bridge }\end{array}$ & $\begin{array}{c}\text { Direction } \\
2 \text { : } \\
\text { from } \\
\text { Gellert } \\
\text { sqr }\end{array}$ & $\begin{array}{l}\text { Direction } \\
\text { 3: } \\
\text { from } \\
\text { Irinyi J. } \\
\text { str }\end{array}$ & $\begin{array}{c}\text { Direction } \\
4: \\
\text { from } \\
\text { Egry J. } \\
\text { str }\end{array}$ \\
\hline $\begin{array}{l}\text { Travel } \\
\text { time } \\
\text { delay cost } \\
\text { of current } \\
\text { signalling } \\
\text { [HUF/h] }\end{array}$ & $\begin{array}{l}\text { Passenger } \\
\text { Car }\end{array}$ & 58204 & 64544 & 37295 & 25481 \\
\hline $\begin{array}{l}\text { Travel } \\
\text { time } \\
\text { delay cost } \\
\text { of new } \\
\text { signalling } \\
\text { [HUF/h] }\end{array}$ & $\begin{array}{l}\text { Passenger } \\
\text { Car } \\
\text { total sum }\end{array}$ & 32472 & 35587 & 54782 & 36853 \\
\hline
\end{tabular}

Tab. 11. The current and optimal (minimized environmental pollution) green times

\begin{tabular}{|c|c|c|c|c|}
\hline & $\begin{array}{c}\text { Direction } \\
1: \\
\text { from } \\
\text { Petőfi } \\
\text { bridge }\end{array}$ & $\begin{array}{c}\text { Direction } \\
2: \\
\text { from } \\
\text { Gellert } \\
\text { sqr }\end{array}$ & $\begin{array}{c}\text { Direction } \\
3: \\
\text { from } \\
\text { Irinyi J. } \\
\text { str }\end{array}$ & $\begin{array}{c}\text { Direction } \\
4: \\
\text { from } \\
\text { Egry J. } \\
\text { str }\end{array}$ \\
\hline $\begin{array}{l}\text { Current green } \\
\text { time [s] }\end{array}$ & 33 & 34 & 19 & 14 \\
\hline $\begin{array}{l}\text { Theore-tical case of } \\
\text { minimal delays }[\mathrm{s}]^{*}\end{array}$ & 45 & 46 & 7 & 2 \\
\hline $\begin{array}{l}\text { Optimal in case } \\
\text { of minimal delays [s] }\end{array}$ & 42 & 43 & 10 & 5 \\
\hline
\end{tabular}




\begin{tabular}{|c|c|c|c|c|c|}
\hline & & $\begin{array}{c}\text { Direction } \\
1: \\
\text { from } \\
\text { Petófi } \\
\text { bridge }\end{array}$ & $\begin{array}{c}\text { Direction } \\
2: \\
\text { from } \\
\text { Gellert } \\
\text { sqr }\end{array}$ & $\begin{array}{l}\text { Direction } \\
3: \\
\text { from } \\
\text { Irinyi J. } \\
\text { str }\end{array}$ & $\begin{array}{c}\text { Direction } \\
4: \\
\text { from } \\
\text { Egry J. } \\
\text { str }\end{array}$ \\
\hline \multirow{4}{*}{$\begin{array}{l}\text { Environ- } \\
\text { mental } \\
\text { emission } \\
\text { cost of } \\
\text { current } \\
\text { signalling } \\
\text { [HUF/h] }\end{array}$} & $\begin{array}{c}\text { Passenger } \\
\text { Car }\end{array}$ & 156.6 & 136.59 & 60.9 & 89.61 \\
\hline & $\begin{array}{l}\text { Goods } \\
\text { Vehicle }\end{array}$ & 4.35 & 16.53 & 4.06 & 4.35 \\
\hline & $\begin{array}{l}\text { sum of } \\
\text { direction }\end{array}$ & 160.95 & 153.41 & 65.25 & 94.25 \\
\hline & total sum & \multicolumn{4}{|c|}{437.57 .86} \\
\hline \multirow{4}{*}{$\begin{array}{l}\text { Environ- } \\
\text { mental } \\
\text { emission } \\
\text { cost of } \\
\text { new } \\
\text { signalling } \\
\text { [HUF/h] }\end{array}$} & $\begin{array}{l}\text { Passenger } \\
\text { Car }\end{array}$ & 86.42 & 76.27 & 88.16 & 131.95 \\
\hline & $\begin{array}{l}\text { Goods } \\
\text { Vehicle }\end{array}$ & 2.32 & 9.28 & 6.09 & 6.38 \\
\hline & $\begin{array}{l}\text { sum of } \\
\text { direction }\end{array}$ & 88.74 & 85.55 & 94.25 & 138.33 \\
\hline & total sum & \multicolumn{4}{|c|}{406.87} \\
\hline \multirow{2}{*}{$\begin{array}{c}\text { Change } \\
{[\%]}\end{array}$} & $\begin{array}{l}\text { sum of } \\
\text { direction }\end{array}$ & $-44.86 \%$ & $-44.21 \%$ & $44.63 \%$ & $46.89 \%$ \\
\hline & total sum & \multicolumn{4}{|c|}{$-14.10 \%$} \\
\hline
\end{tabular}

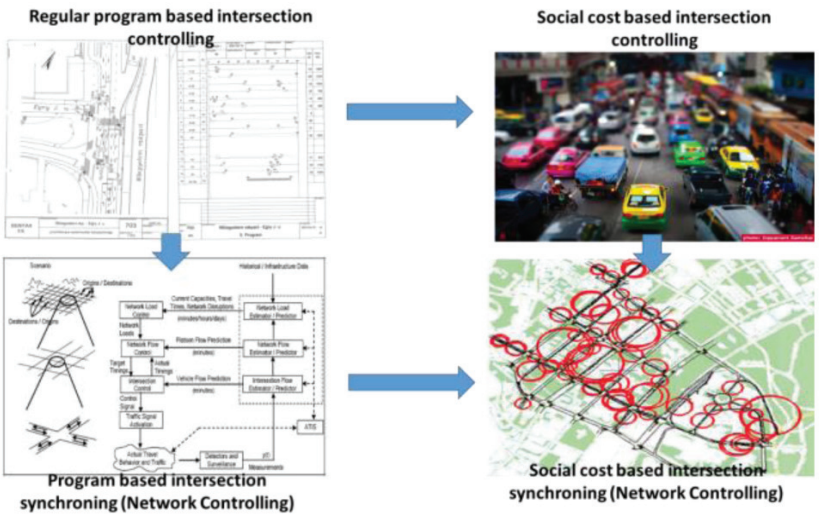

Fig. 4. Hierarchy structure of intersection controls

\section{Acknowledgements}

The authors are grateful to the support of Bólyai János Research fellowship of HAS (Hungarian Academy of Science). The authors are grateful for the support of Prof. Dr. Florian Heinitz, Director of Transport, and Spatial Planning Institute in Erfurt, Germany. Further on the authors are grateful for the support of David Hajnal MSc student for his valuable contribution.

\section{References}

1 Török Á., Berta T., Travel time reduction due to infrastructure development in Hungary. PROMET - Traffic\&Transportation, 22(1), 23-28 (2010). DOI: $10.7307 /$ ptt.v22i1.161

2 Makaras R., Sapragonas J., Keršys A., Pukalskas S., Dynamic model of a vehicle moving in the urban area. Transport, 26(1), 35-42 (2011). DOI: $\underline{10.3846 / 16484142.2011 .558630}$

3 Wang H., Fu L., Zhou Y., Li H., Modelling of thefuel consumption for passenger cars regarding driving characteristics. Transport Research Part D, 13(7), 479-482 (2008).

DOI: $10.1016 / j . \operatorname{trd} .2008 .09 .002$

4 Szendrő G., Csete M., Török Á., Unbridgeable gap between transport policy and practice in Hungary. Journal Of Environmental Engineering And Landscape Management, 20(2), 104-109 (2012). DOI: $10.3846 / 16486897.2012 .660881$

5 Smit R., Brown A.L., Chan Y.C., Do air pollution emissions and fuel consumption models for roadways include the effects of congestion in the roadway traffic flow? Environmental Modelling \& Software, 23(10-11), 1262-1270 (2008). DOI: 10.1016/j.envsoft.2008.03.001

6 Jakimavičius M., Burinskienè M., Route planning methodology of an advanced traveller information system in Vilnius city. Transport, 25(2), 171-177 (2010).

DOI: $10.3846 /$ transport.2010.21
7 Ren H., Liu H., Long J., Gao Z., Dynamic user optimal signal design at isolated intersections. PROMET - Traffic\&Transportation, 25(1), 13-22 (2013).

DOI: $10.7307 /$ ptt.v25i1.1243

8 Bogdanović V., Ruškić N., Papić Z., Simeunović M., The research of vehicle acceleration at signalized intersections. PROMET - Traffic\&Transportation, 25(1), 33-42 (2013). DOI: $\underline{10.7307 / p t t . v 25 i 1.1245}$

9 Mészáros F., Markovits-Somogyi R., Bokor Z., Modelling and multi-criteria optimization of road traffic flows considering social and economic aspects. Scientific Journal on Transport and Logistics, 3(1), 70-82 (2012).

10 Török Á., Siposs Á., Mészáros F. The history and the foreseeable future of road tolling in Hungary. PROMET - Traffic\&Transportation, 23(5), 389-396 (2011).

DOI: $10.7307 /$ ptt.v23i5.157

11 Junevičius R., Bogdevičius M., Torok A., Modelling of internal combustion engines'emission through the use of traffic flow mathematical models. Transport, 26(3), 271-278 (2011). DOI: $10.3846 / 16484142.2011 .621978$

12 Csikos A., Varga I., Real-time estimation of emissions emerging from motorways based on macroscopic traffic data. Acta Polytechnica Hungarica, 8(6), 95-110 (2011).

13 Zöldy M., Ethanol-biodiesel-diesel blends as a diesel extender option on compression ignition engines. Transport, 26(3), 303-309 (2011). DOI: $\underline{10.3846 / 16484142.2011 .623824}$ 
14 Bereczky Á., Parameter analysis of NO emissions from spark ignition engines. Transport, 27(1), 34-39 (2012).

DOI: $10.3846 / 16484142.2012 .664563$

15 Barabas I., Todorut I. A., Predicting the temperature dependent viscosity of biodiesel-diesel-bioethanol blends. Energy \& Fuels, 15(12), 5767-5774 (2011).

DOI: $\underline{10.1021 / \mathrm{ef} 2007936}$

16 Negoițescu A., Tokar A., Investigations on car emissions under the urban traffic conditions with the influence on Timişoara air quality. Transport, 28(1), 38-45 (2013).

DOI: $10.3846 / 16484142.2013 .781060$

17 Makarevičienè V., Matijošius J., Pukalskas S., Vègneris R, Kazanceva I., Kazancev K., The exploitation and environmental characteristics of diesel fuel containing rapeseed butyl esters. Transport, 28(2), 158-165 (2013).

DOI: $10.3846 / 16484142.2013 .801364$
18 Gál G., Determining and comparing the qualitative consistency of urban and highway traffic flows. Periodica Polytechnica Transportation Engineering, 40(2), 61-65 (2012). DOI: $10.3311 /$ pp.tr.2012-2.03

19 Tánczos L., Bokor Z., Practical adaptation opportunities of social cost based transport pricing systems (in Hungarian). Scientific Review of Transport, 54(5), 185-192 (2004). 